\title{
Editorial
}

\section{Feasibility of route extrapolation in risk assessment}

For the protection of public health it is often necessary to estimate acceptable concentrations of chemicals present in the environment when data are lacking for the appropriate route of human exposure. The use of uncertainty factors to protect against potential differences in potency between routes of administration may, however, lead to an overly restrictive risk characterisation or regulation or both. On the other hand, there may be cases when the uncertainty factors are inadequate to protect human health. To minimise such errors, and to limit the size of uncertainty factors necessary, every effort should be made to determine the relation between administered dose and concentration of the active agent at the critical target site when carrying out route extrapolation.

Extrapolation is most commonly carried out from the intragastric to the inhalation route and vice versa, although many human exposures occur primarily through the dermal route. While other exposure routes such as intravenous infusion and subcutaneous injection are used for therapeutic agents or in experimental animal studies, they are not the common means of environmental exposure to toxic agents. It is none the less necessary, at times, to extrapolate from experiments on animals using such routes if no other data are available. The methods commonly used involve conversion of the concentration in each medium (air and water, for example) to a dose per unit body weight basis, using either estimated or measured values for respiratory minute volume and food or water intake. Adjustments are often made for absorption efficiency if data are available. Unfortunately, other toxicokinetic factors are seldom taken into account during route extrapolation.

One of the earlier proposals for route extrapolation was outlined by Stokinger and Woodward. ${ }^{1}$ They suggested that threshold limit values (TLVs) set by the American Council of Government Industrial Hygienists for permissible levels of substances in workroom air could be used to calculate the equivalent allowable doses swallowed in drinking water. In essence, the total pulmonary uptake was calculated by multiplying the allowable concentration in the air by the amount of air exchanged in the lungs over an eight hour period, and dividing by two, to account for an assumed $50 \%$ absorption of the inhaled agent. This amount was considered to be an allowable level present in two litres of drinking water drunk by an "average" person. Absorption from water was assumed to be complete unless data were available to indicate otherwise.

One of the specific criticisms of the Stokinger and Woodward method ${ }^{1}$ was that many TLVs are based on irritant action or organoleptic effects, neither of which have relevance to systemic toxic effects. Stokinger and Woodward recognised the potential error in such an extrapolation and therefore proposed that an uncertainty factor ranging from 10 to 100 be incorporated into the determination of allowable intakes involving route extrapolation. The assumption of $50 \%$ absorption via inhalation versus $100 \%$ by the oral route could be considered to be an additional uncertainty factor if extrapolation is from the inhalation to the oral route.

Pepelko and Withey compared published values of $\mathrm{LC}_{50}$ and $\mathrm{LD}_{50}$ for 49 chemicals. ${ }^{2}$ After conversion of the inhaled concentration to a dose per unit body weight, the oral to inhalation ratios were found to vary from $0 \cdot 1$ to 55 . On the basis of these acute toxicity comparisons, with mortality as the end point, the uncertainty factors proposed by Stokinger and Woodward $^{1}$ would be adequate, in most cases, to account for the uncertainty in route extrapolation, at least for the chemicals examined. On the other hand, risk would be overestimated to a considerable degree in some instances. Unfortunately, similar comparisons after chronic exposure are available for only a few chemicals.

Even if an adequate chronic toxicology study is available, however, large errors can occur when attempting route extrapolation, because potency may be influenced by several route specific factors. One such factor is absorption efficiency. Cells lining the intestinal tract may show differing permeability to certain chemicals than those in the lung. Active uptake mechanisms may also differ between routes. For particulate matter of limited solubility, long residence time in the lung may allow considerable solubilisation and absorption to occur, whereas little 
uptake may take place from the gastrointestinal tract due to rapid passage. Furthermore, uptake by pulmonary macrophages can greatly increase the rate of particle solubilisation. ${ }^{3}$

Another factor is the possibility of chemical transformation before or during absorption. Dollery et al have shown that here is a 1000 -fold reduction in systemic availability of is proterenol when it is administered by mouth as compared with intravenously, due to formation of the inactive ethereal sulphate across the gut wall. ${ }^{4}$ Metabolism by intestinal organisms can result in either activation or inactivation of ingested chemicals. Pulmonary macrophages also have metabolic capability. For example, benzo(a) pyrene coated on particles is metabolised to its proximate carcinogenic form by pulmonary macrophages. ${ }^{5}$

Passage through certain organs may result in the activation or inactivation of an agent before reaching the target site-that is, "first pass" effects may occur. The liver plays a major part in first pass effects since it contains the largest volume of metabolising enzymes and since most chemicals absorbed through the gastrointestinal tract pass through the liver before entering the systemic circulation. For example, organic nitrates have been shown to be completely degraded to inactive metabolites with one pass through the liver. ${ }^{6}$ The lung may also be considered a first pass organ since all inhaled chemicals must pass through it before entering the peripheral circulation and Roth and Wiersma listed 22 agents acted on by the lung. ${ }^{7}$ Xenobiotic metabolism may even occur during dermal absorption. Since the lung, liver, or skin do not necessarily metabolise the same chemicals, the amount of active metabolite entering the systemic circulation can vary widely. In some cases the agent may be highly toxic by one route and without effect by another.

Route related differences in effectiveness of the agent may occur in chemicals with short half lives. In such cases intermittent exposure, such as that occurring with ingestion, may result in large fluctuations in concentration at the target organ. During inhalation exposure, concentrations of such chemicals in the blood tend to be stable once equilibrium is reached. Some periodic dosing regimens may result in acute toxic effects, whereas blood concentration remains at subthreshold levels when the same total dose is administered at a steady rate over the same period. For example, Evans reported that guinea pigs could survive 20 times the minimal lethal dose of hydrogen cyanide when it was administered at a steady rate over a 67 minute period. ${ }^{8}$

For chemicals with limited blood solubility, the concentration time relations in the blood are also likely to differ with exposure route even though the area under the time concentration curve may be the same. During inhalation exposure, uptake from the lungs will be rapid until the blood concentration reaches a level dependent on the blood to air partition coefficient of the chemical, the concentration in the inspired air, and the rate of elimination. Absorption? then slows to a rate sufficient to replace the amount lost by metabolism, excretion, or storage. Greate uptake can take place from the liquid media in the gastrointestinal tract since absorption is not limite $\$$ by the partition coefficient, resulting in higher liveg concentrations. On the other hand, since the inspired air concentration is zero during oral exposure, the blood to air partition coefficient will be exceeded and considerable elimination will occur during passage of blood through the lungs. Thus oral exposure to thesळ types of chemicals results in not only greate $\vec{p}$ fluctuation in overall blood concentration that occurs during inhalation exposure but in greater con $\overrightarrow{0}$ centration in the liver than in other organs.

If the critical effect occurs at a portal of entry fote one route the critical site of action, the effective dose and even the nature of the effect will probably differ for another route of exposure. Paraquat is one of the few chemicals for which the lung appears to be the critical target organ irrespective of the route og exposure. ${ }^{9}$ Portal of entry effects are likely if the com ${ }^{-}$ pound is either highly reactive, such as formaldehyde deposited in the nasal cavity, ${ }^{10}$ or if it has a long idence time at the portal of entry-for example, pare ticulate matter of limited solubility deposited in lung.

Each of the factors discussed involves, in some way, the temporal relation between administered dos and concentration of the active agent at the critica target organ. If information regarding this relation if available for both routes of exposure, along with an adequate chronic toxicology study, by one route extrapolation may be carried out with only a smaH degree of uncertainty. Since such information is sete dom available, however, the reliability of extrapo lation depends on the degree of knowledge concerning the previously discussed properties of the agent. Extrapolation is reasonable if absorption efficiency is the same among routes, or differs by a known degree; if half life of the chemical is long, prefo erably greater than 24 hours; if first pass effects are minimal; if there is no significant chemical trans? formation by intestinal microflora or by pulmonarow macrophages; if the critical target tissue is not at the portal of entry; if the chemical is relatively soluble ig body fluids; and if an adequate chronic toxicity iso available for the route used as a basis for extrapo? lation.

While route to route extrapolation may still be feaథ sible if one or more of these conditions are absent, of if knowledge concerning their presence is lacking, the 
reliability in the extrapolation is decreased. This results in the necessity for larger uncertainty factors. If minimal data regarding these conditions are unavailable then route extrapolation cannot be justified.

In summary, route extrapolation can usually be carried out with a reasonable degree of confidence if care is taken to consider the potential sources of error discussed above. It should also be recognised that the degree of uncertainty will vary depending on the presence, or absence, of certain properties of the agent and the degree to which these properties can be accounted for.

US Environmental Protection Agency,

W E PEPELKO

Carcinogen Assessment Group,

Office of Health and Environmental Assessment

(RD-689),

Washington, DC 20460, USA.

\section{References}

1 Stokinger HE, Woodward RL. Toxicological methods for establishing drinking water standards. Journal of the American Water Works Association 1958;50:515-29.

2 Pepelko WE, Withey JR. Methods for route-to-route extrapolation of dose. Toxicology and Industrial Health 1986;1:153-75.

3 Andre S, Metvier H, Lantenos G, Masse R. In: Proceedings of inhalation toxicology. The design and interpretation of inhalation studies and their use in risk assessment. 1987. Hanover: Springer-Verlag (in press).

4 Dollery CT, Davis DS, Conolly ME. Differences in the metabolism of drugs depending on their route of administration. Ann NY Acad Sci 1971;179:108-14.

5 Harris CC, Hsu IC, Stoner GD, Trump BF, Selkirk JK. Human pulmonary macrophages metabolize benzo(a)pyrene to proximate and ultimate carcinogens. Nature 1978;272:633-4.

6 Needleman P, Lang S, Johnson EM Jr. Organic nitrates: Relationship between biotransformation and rational angina pectoris therapy. J Pharmacol Exp Ther 1972;181:489-97.

7 Roth RA, Wiersma DA. Role of the lung in total body clearance of circulating drugs. Clin Pharmacokinet 1979;4:355-67.

8 Evans CL. The toxicity of hydrogen sulphide and other sulphides. $Q J$ Exp Physiol 1967;52:231-48.

9 Reinhardt CF, Brittelli MR. Heterocyclic and miscellaneous nitrogen compounds. In: Clayton GD, Clayton FE, eds. Industrial hygiene and toxicology. Vol 2A. New York: John Wiley and Sons, 1981:2671-82.

10 Chang JFC, Gross EA, Swenberg JA, Barrow CS. Nasal cavity deposition, histopathology, and cell proliferation after single or repeated formaldehyde exposures in $B 6$ or $F_{1}$ mice and F-344 rats. Toxicol Appl Pharmacol 1983;65:161-76.

\section{Correspondence and editorials}

The British Journal of Industrial Medicine welcomes correspondence relating to any of the material appearing in the journal. Results from preliminary or small scale studies may also be published in the correspondence column if this seems appropriate. Letters should be not more than 500 words in length and contain a minimum of references. Tables and figures should be kept to an absolute minimum. Letters are accepted on the understanding that they may be subject to editorial revision and shortening.

The journal now also publishes editorials which are normally specially commissioned. The Editor welcomes suggestions regarding suitable topics; those wishing to submit an editorial, however, should do so only after discussion with the Editor. 